\title{
ASSESSMENT OF SHIELDING EFFECTIVENESS AND Percolation Threshold OF COMPOSite MATERials
}

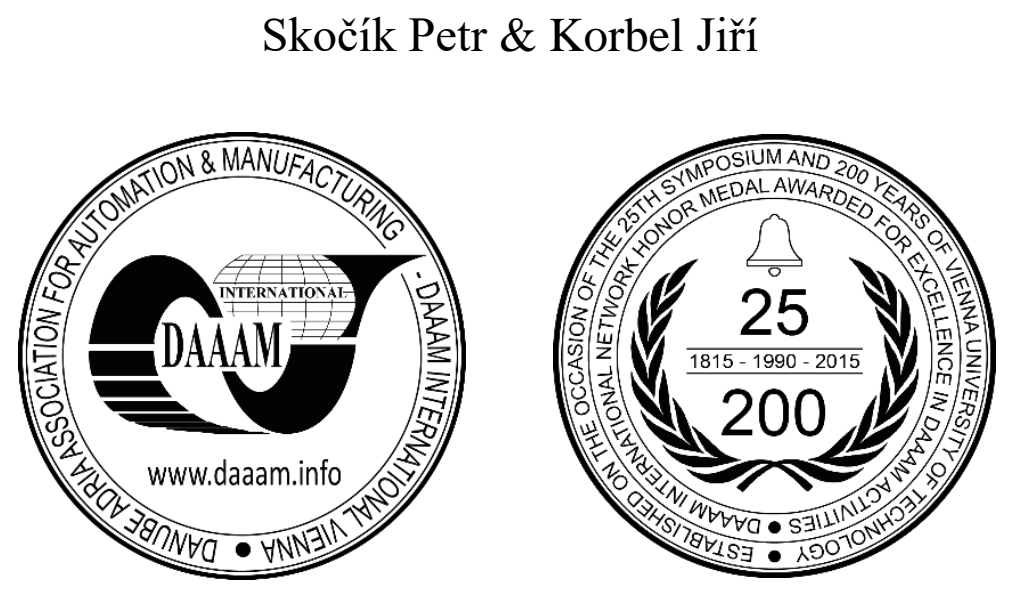

This Publication has to be referred as: Skocik, P[etr] \& Korbel, J[iri] (2017). Assessment of Shielding Effectiveness and Percolation Threshold of Composite Materials, Proceedings of the 28th DAAAM International Symposium, pp.00540062, B. Katalinic (Ed.), Published by DAAAM International, ISBN 978-3-902734-11-2, ISSN 1726-9679, Vienna, Austria

DOI: $10.2507 / 28$ th.daaam.proceedings.007

\begin{abstract}
This paper deals with the protection of avionics of small airplanes in an electromagnetic environment. Various composite materials are used for this purpose. The mechanical properties of these materials are very well described. On the other hand, the electrical properties are described insufficiently. A fabric with long copper-plated polyamide fibers was selected as a suitable shielding material. The aim is to verify the shielding effectiveness of the fabric by measuring the transverse electromagnetic wave on the coaxial line. Further, determine the flat fiber concentration required to achieve a certain shielding efficiency of given composite sample and its percolation threshold which is an important parameter determining the conductivity of the composite material. It is therefore possible to determine the critical content of the filler in which the composite material starts to act as a conductor.
\end{abstract}

Keywords: Shielding effectiveness; Percolation threshold; Composite materials; Coaxial line

\section{Introduction}

The development of society brings new demands in all areas of human research. At present, considerable progress has been made in the field of information technology, which is related to developments in the field of microelectronics, computing and control technology. Unfortunately, such development also has its negatives. One of the notable problems is the reliability of these devices. This is influenced not only by the quality of production itself but also by external influences on the equipment. At present, this problem escalates as the environment is overwhelmed by a variety of interfering signals. These signals may have an undesirable effect on any device resulting in technological failures or accidents. Typical representatives of such devices are, for example, devices used in air traffic control or the aerospace industry itself. It is therefore a prerequisite to prevent possible failure of a device during its design and construction, ie to design the equipment in a way that it can operate properly even in the environment with any source of electromagnetic interference. The development of interference resistant devices is noticeable especially since the nineties. Of course, this has economic consequences as well. For example, designing the aircraft without consideration of the electromagnetic interference can lead to failure in the safety test during the final certification process, including the measurement of 
avionics resistance to electromagnetic interference. Which implies the return of the prototype back to the initial phase of development with all associated costs.

A significant part of today's airplanes is made of composite materials. The aim of these materials is to achieve a lighter and more durable design with longer life and reliability. These materials are very well described in terms of mechanical properties, but unfortunately only at least in terms of electrical properties. This work is focused on verifying the shielding efficiency of a suitable composite material and to determine its percolation threshold which is an important parameter determining the conductivity of the composite material. It defines the content of the filler in which the composite starts to act as a conductor.

\section{Electromagnetic compatibility}

Electromagnetic Compatibility (EMC) is defined as the ability of a device or system to perform properly in an environment where other sources of electromagnetic signals (natural or artificial) operate. Additionaly, these devices or systems must not radiate signals that are interfering with other equipment [5]. EMC has two main areas, as can be seen in Figure 1.

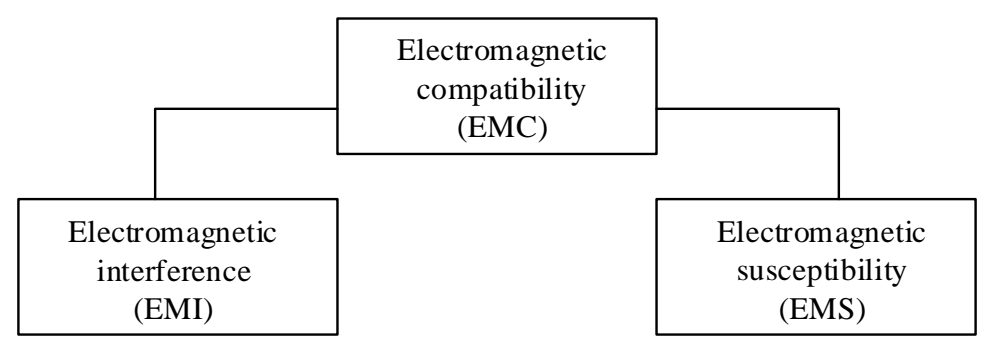

Fig. 1. Main areas of the EMC

Electromagnetic interference (EMI) is the process by which the signal generated by a source of interference is transmitted through electromagnetic coupling to interfering systems. It deals with identification of interference sources, describtion and measurement of interfering signals and identification of parasitic transmission paths. Compatibility of the whole system is achieved by technical measures, especially on the side of the sources of interference and their transmission paths. The EMI mainly concerns the causes of interference and its removal [1].

Electromagnetic susceptibility (EMS) is the ability of a device or system to operate without failures in an environment where electromagnetic interference occurs. EMS is therefore focused on technical measures that increase the electromagnetic immunity to interference disturbances at the receiver. The EMS thus concerns with elimination of the interference consequences without removing its causes [1].

\section{Electromagnetic shielding}

Electromagnetic shielding is one of the means of electromagnetic compatibility to reduce the emission-side of interference sources and to increase electromagnetic immunity on the receiver side. Shielding from a technical point of view is a structural means (shielding cover) for weakening the field of disturbing signals. It is used to protect electrical and electronic components, devices or systems that can be both sources and receivers of electromagnetic interference. It is one of the highly efficient ways of electromagnetic protection against power interference [3], [5].

The shielding itself is defined as the ratio of the intensity of the electromagnetic field in front of and behind the shielding material. A prerequisite is that the shielding material is an infinite plane located between the source and the receiver of the electromagnetic radiation. The region around the source of electromagnetic radiation can be divided into three areas [3]:

- distant field - field properties depend on the environment in which the wave propagates,

- near field - field properties depend primarily on the properties of sources of electromagnetic interference and the surroundings,

- transition area - located between the previous two areas.

The theoretical boundary between the distant and near field is assumed at $r=\lambda / 2 \pi$ (approximately $\lambda / \pi$ from wavelength $\lambda$ ). In practice, when talking about a distant field, the distance $r>5 \lambda / 2 \pi$ is assumed. If the shielding material is in the near field, the distance of the wave source from the shielding material is much smaller than the wavelength $\lambda$, more precisely $r<<\lambda / 2 \pi$ [3]. 


\subsection{Electromagnetic shielding efficiency}

The effect of electromagnetic shielding as a linear system can be characterized by the Shielding Effectivity (SE), which is defined by the ratio of the electric field intensity $E_{t}$ or the magnetic field intensity $H_{t}$ at a certain point of the shielded space to the intensity $E_{i}\left(H_{i}\right)$ of the field impacting on the shielding material or bulkhead [5]. The logarithmic SE rate is determined by [1] as:

$$
\mathrm{SE}=20 \cdot \log \left|\frac{\mathrm{E}_{\mathrm{t}}}{\mathrm{E}_{\mathrm{i}}}\right|=20 \cdot \log \left|\frac{\mathrm{H}_{\mathrm{t}}}{\mathrm{H}_{\mathrm{i}}}\right| \quad[d B]
$$

If the incident wave is homogeneous and the environment on both sides of the shielding bulkhead is the same, both definitions SE (1) are the same, since the $E$ and $H$ fields are mutually bound by the same impedance of the environment. Otherwise, the equation (1) give different results [5].

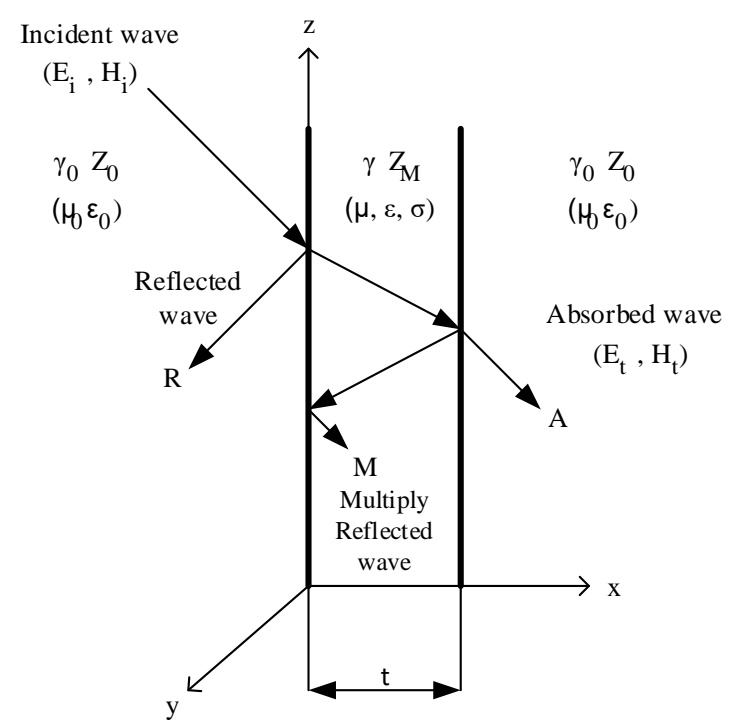

Fig. 2. The physics of the shielding effectiveness [3], [5]

Calculating the SE in Figure 2 needs to determine the components of the electromagnetic wave penetrating the shielded area at a known magnitude of the incident wave $E_{i}, H_{i}$, known parameters of the shielding bulkhead $(\varepsilon, \mu, \sigma, \mathrm{t})$ and known parameters of the surrounding environment $\left(\varepsilon_{0}, \mu_{0}\right)$. The solution is based on Maxwell's equations and after mathematical modifications it can be obtained as [1], [5]:

$$
S E=20 \cdot \log \mid \frac{\left(Z_{0}+Z_{M}\right)^{2}}{4 Z_{0} Z_{M}} \cdot e^{\gamma t} \cdot\left[1-\left(\frac{Z_{0}-Z_{M}}{Z_{0}+Z_{M}}\right)^{2} \cdot e^{-2 \gamma t}\right] \quad[d B]
$$

$Z_{0}$ is the impedance of the free environment before and behind the shielding bulkhead and $Z_{M}$ id the impedance of the conductive environment of the bulkhead. For these parameters applies [5]

$$
Z_{0}=\sqrt{\frac{\mu_{0}}{\varepsilon_{0}}} \quad[\Omega] \quad \text { and } \quad Z_{M}=\sqrt{\frac{j \omega \mu}{\sigma}} \quad[\Omega]
$$

The parameter $\gamma$ expresses the propagation constant of planar electromagnetic wave in the conductive environment of the shielding bulkhead [5]

$$
\gamma=\sqrt{j \omega \mu \sigma}=(1+j) \cdot \sqrt{\frac{\omega \mu \sigma}{2}}=a+j \beta
$$


The relation (2) for the SE in the distant electromagnetic field can be rewritten to the form which better describes the physical principle of the shielding efficiency presented in Fig. 2 [1], [5]:

$$
S E=R+A+M \quad[d B]
$$

where $R$ represents attenuation caused by reflection, $A$ describes the attenuation caused by absorbtion of part of the energy of the electromagnetic wave when passing through the shielding bulkhead, and $M$ represents the multiple reflections that occur within the bulkhead. Reflection and absorbtion are most important for determining overall shielding efficiency, as can be seen in Fig. 2.

\section{Verification of the shielding efficiency of the selected composite material}

Composite materials are heterogeneous materials composed of two or more phases that differ significantly from each other by their mechanical, physical and chemical properties. The composite materials are produced by mechanical mixing of the individual components. This differs, for example, from alloys which are also heterogeneous [6]. Composite materials are characterized by synergism, which means that the properties of the composite material are higher than would correspond to mere proportional summation of the properties of the individual components. The existence of synergism is very important as it leads to the acquisition of new features. A typical example of synergistic behavior is a composite material composed of a ceramic matrix reinforced by ceramic fibers. Although both the matrix and the fibers are individually very fragile, the resulting composite material is characterized by a certain degree of toughness. The spreading fracture of the composite material is inhibited at the matrix and fiber interface. There is a deflection of the crack propagation direction and the intense mutual friction between the matrix and the pulling fibers. The quality of the interface between the matrix and the reinforcement therefore has a major effect on the properties of the resulting composite material [6], [11].

Composite material was selected from the three available samples of metallized fabric shown in Figure 3 . The selection was made on the assumption that the sample would be used and would serve as a suitable shielding material [12]. This is a copper-plated polyamide fiber fabric labeled PBN-II-1.3-Cu-Sn (see Figure 3) by the Hungarian firm LORIX [8]. The metallized layer is very thin, usually around $0.1 \mu \mathrm{m}$.

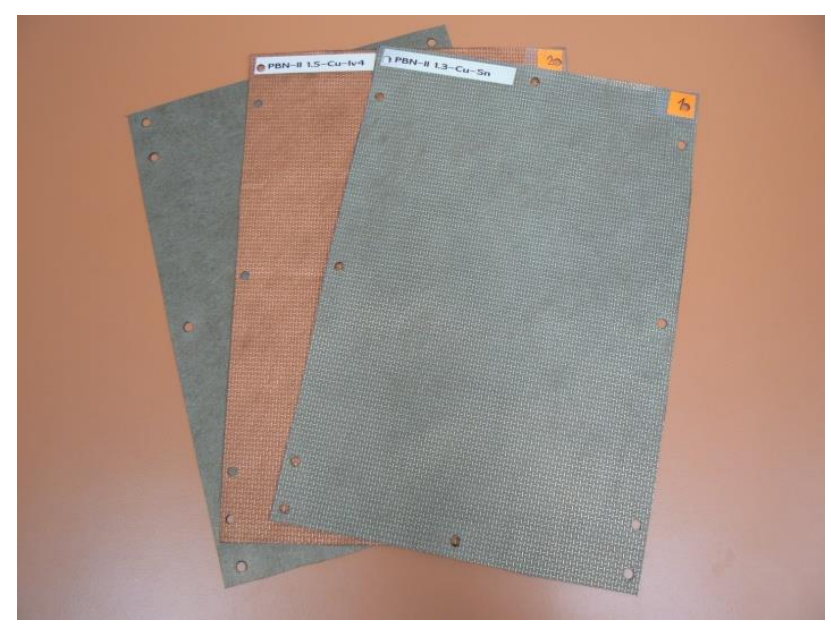

Fig. 3. Available samples of composite material

Measurements of the sample were carried out on a co-axial line supporting Transverse electromagnetic mode (TEM). The inlet and outlet sections of the line connect an annular flange through the adapter. The adapter consists of two symmetrical parts. The body of the adapter, including the flange, was made of brass and the parameters were designed with respect to the $50 \Omega$ input and output impedance of the line. The coaxial line description is given in [3] and [7]. Configuration of the assembly is shown in Figure 4.

The measuring assembly consists of the coaxial line, the SMR20 signal generator (in Figure 4, bottom right) and the spectrophotometer FSP40, both from Rohde \& Schwarz. The measured sample was clamped into a stainless steel collar specially designed and manufactured to minimize unintentional signal flow from the generator to the analyzer. Nevertheless, it is necessary to consider the integrity of the measured sample of the fabric and hence the occurring gaps between the fibers. The whole measurement process has been automated using the laptop and Agilent VEE Pro 7.5 software. It is a graphical development environment with an open structure allowing to work with hardware and software from almost any manufacturer.

Measurement of shielding efficiency of the sample was performed over a frequency range of 1 to $10 \mathrm{GHz}$. The resulting graphical dependence is shown in Figure 5. 


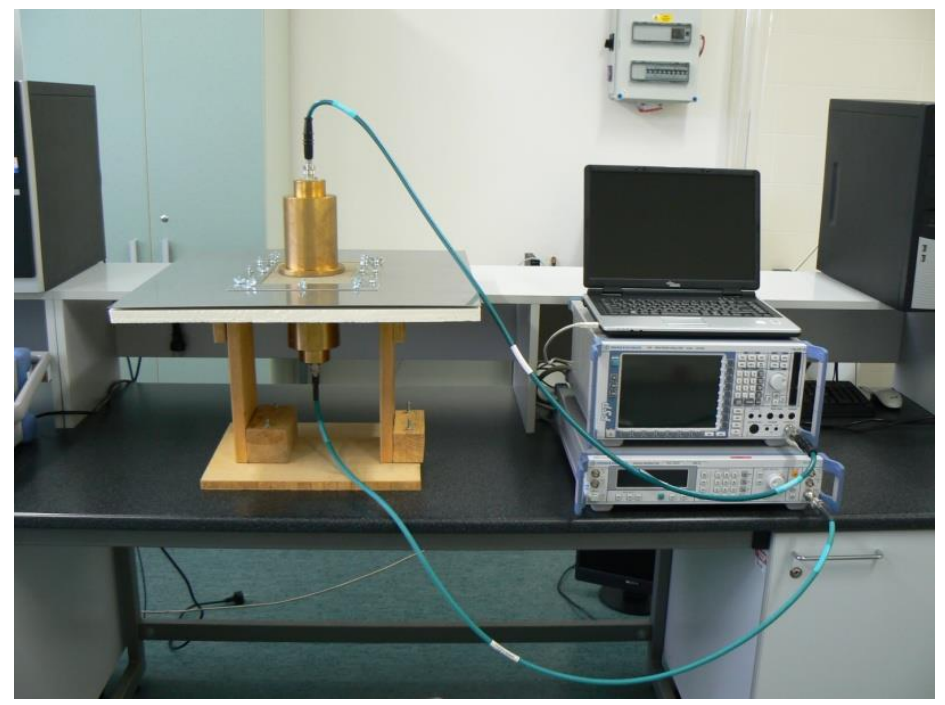

Fig. 4. Configuration of the measuring device

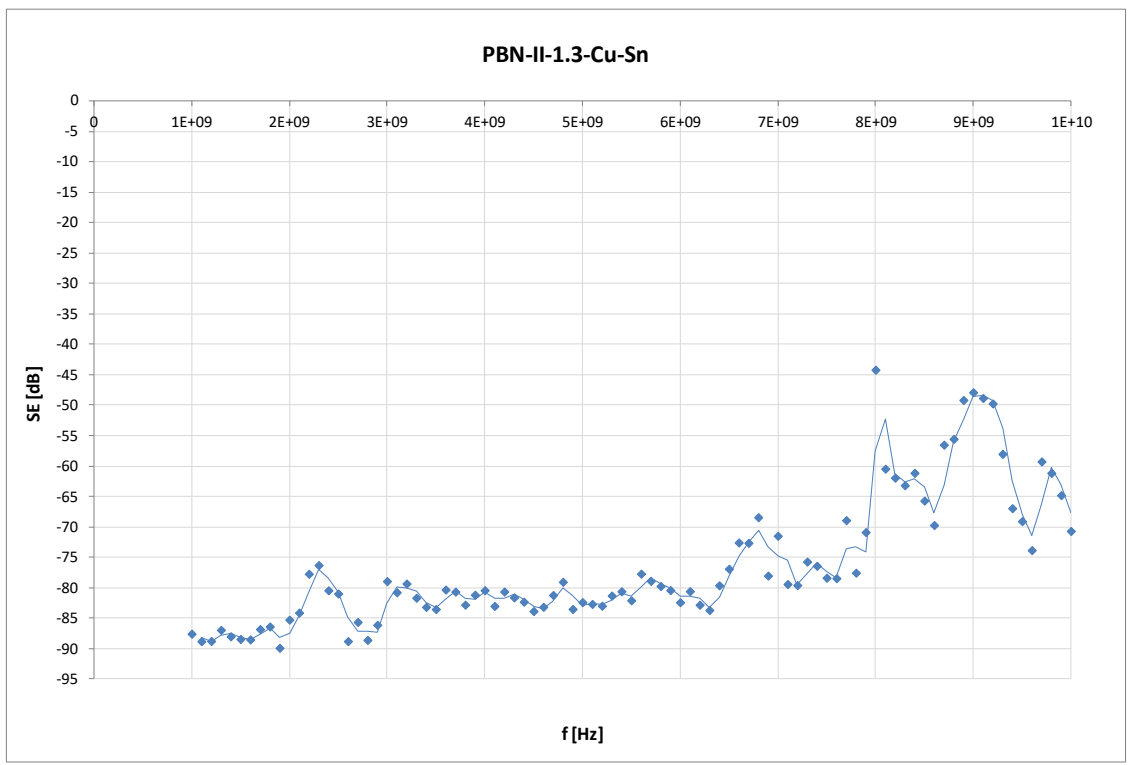

Fig. 5. Shielding efficiency of PBN-II-1.3-Cu-Sn

The comparison of the sample SE values supplied by the manufacturer and obtained by the measurement is performed only for $1 \mathrm{GHz}$ frequency because the measurement was performed for higher frequencies than $1 \mathrm{GHz}$. It can be seen from Figure 5 that the measured SE at a given frequency is about $-87 \mathrm{~dB}$. From the data supplied by the manufacturer, the $\mathrm{SE}$ value for $1 \mathrm{GHz}$ can be determined to about $-75 \mathrm{~dB}$ [8]. The difference between the values is obvious. By verification, the SE of the given sample was found to be higher than the manufacturer states, which may not be a false result. On the contrary, it may be an indication of the real SE of the given sample. It can be assumed that the manufacturer gives the minimum guaranteed SE of the sample or the possibility that the sample may have a higher metallization thickness.

\section{Determination of the percolation threshold of the composite material}

The percolation threshold is an important parameter determining the conductivity of the composite material. It determines the critical content of the filler in which the composite begins to act as a conductor. Corresponding information on the conductivity of the composite material gives a curve, which is often referred to as percolation curve. It is the dependence of the electrical conductivity on the filler concentration and is usually of a sowing shape. At the inflection point of the curve, a critical concentration of the composite filler can be determined. In this point a conductive path is formed, percolation threshold occurs. The relationships between the conductivity and the concentration of the randomly dispersed filler particles are discussed in percolation theory [10], [13].

In the percolation threshold, the number of contacts between the filler particles rises. Further increase in the particle concentration results in a step increase of conductivity up to several orders, especially for low frequencies. If the 
concentration of particles is less than critical, the conductivity of the composite material changes slowly because the distance between the particles is large [13].

To theoretically determine the percolation threshold of the composite sample, it will be based on the simulation of the fabric SE, depending on the surface concentration of the fiber in its structure. The calculation will then determine the performance parameters of $P W R_{T}$ transmission and $P W R_{R}$ reflection. After plotting these parameters into graphical dependence on the fiber concentration in the fabric, the percolation threshold will be estimated. This problem is dealt with in publication of Youngs [4]. A jump change from 0 to 1 for $P W R_{T}$ and from 1 to 0 for $P W R_{R}$ will be sought.

\subsection{Structure of the selected composite}

For the simulation, it was necessary to determine the diameter of the metalized fiber used in the sample and to get an idea of the sample structure itself before the determination of the fiber concentration $S_{V}$ of composite material required to achieve a certain shielding efficiency. For this purpose, a compact stereoscopic microscope Olympus SZX7 with expanded configuration needed for the analysis of the monitored object was used. A complete set of devices is shown in Figure 6.

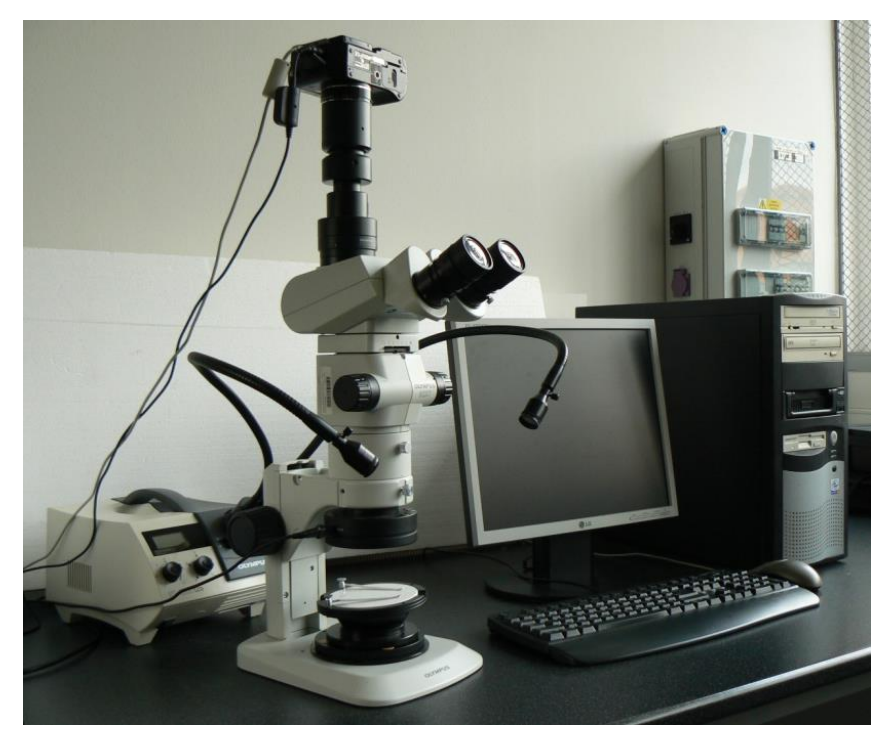

Fig. 6. Configuration of the microscope Olympus SZX7

The image in Figure 7 was used to determine the diameter of the metallised fiber of the fabric. As can be seen in the picture, these are long threads that are limited by sample size. Fibers are stored in several layers of irregular structure and thus their area concentration in the sample differs. From the measured values of the fiber diameters $t$, their mean value and the standard deviation $27,7 \pm 0,7 \mu \mathrm{m}$ were determined. The rounded value of fiber diameter $(28 \mu \mathrm{m})$ was used for the following simulations.

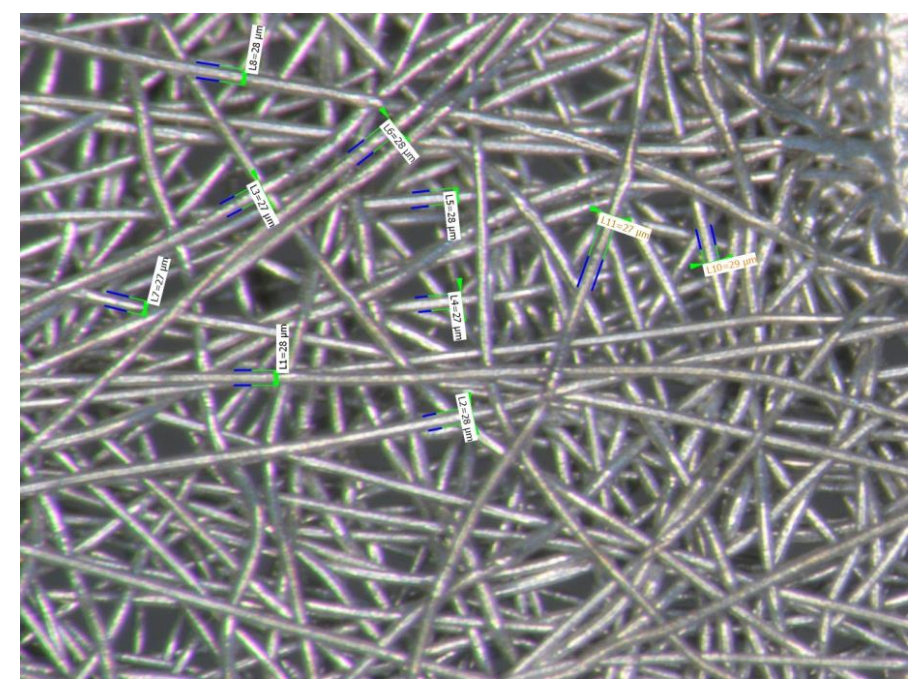

Fig. 7. Image of the fabric structure 


\subsection{Determination of $S_{V}$ to achieve the required $S E$ of the composite material}

Determination of the fiber concentration $S_{V}$ needed to achieve the specific shielding efficiency was done by simulation in the CST Microwave Studio. To simplify the simulations, only the wiregrid structure of the fabric model [2] was considered. It is not a composite structure with several layers of fibers but with only one layer of crossed fibers with a diameter $t=28 \mu \mathrm{m}$. Furthermore, the square-shaped fiber was considered and the results of the simulations were analyzed only for the $1 \mathrm{GHz}$ frequency and the case of the perpendicular electromagnetic wave.

The $S_{V}$ parameter was chosen within the range shown in table 1. It is assumed that the cell size parameter $a=b$.

$$
\begin{aligned}
& S_{V}=\frac{2 t a-t^{2}}{a^{2}} \quad[-] \\
& a=\frac{t}{S_{V}} \cdot\left(1+\sqrt{1-S_{V}}\right) \quad[m]
\end{aligned}
$$

After another parameterization of the sample and verification of all settings, an analysis was performed, from which the SE values for the desired frequency could be read. The SE values obtained from the simulations are shown in table 1. The graphical dependence of SE on the $S_{V}$ fiber concentration in the model structure of the fabric is shown in Fig. 8 .

\begin{tabular}{|c|c|c|c|c|c|}
\hline $\boldsymbol{S}_{\boldsymbol{V}}[-]$ & $\boldsymbol{a}[\mathbf{m m}]$ & $\boldsymbol{a} / \boldsymbol{t}[-]$ & $\boldsymbol{S E}[\mathbf{d B}]$ & $\boldsymbol{P W R _ { \boldsymbol { T } } [ - ]}$ & $\boldsymbol{P W \boldsymbol { R } _ { \boldsymbol { R } } [ - ]}$ \\
\hline 0,8 & 0,051 & 1,809 & -118 & $1,585 \mathrm{E}-12$ & $9,999999999984 \mathrm{E}-01$ \\
\hline 0,7 & 0,062 & 2,211 & -102 & $6,310 \mathrm{E}-11$ & $9,999999999369 \mathrm{E}-01$ \\
\hline 0,6 & 0,076 & 2,721 & -91 & $7,943 \mathrm{E}-10$ & $9,999999992057 \mathrm{E}-01$ \\
\hline 0,5 & 0,096 & 3,414 & -81 & $7,943 \mathrm{E}-09$ & $9,999999920570 \mathrm{E}-01$ \\
\hline 0,4 & 0,124 & 4,436 & -73 & $5,012 \mathrm{E}-08$ & $9,999999498800 \mathrm{E}-01$ \\
\hline 0,3 & 0,171 & 6,122 & -65 & $3,162 \mathrm{E}-07$ & $9,999996838000 \mathrm{E}-01$ \\
\hline 0,2 & 0,265 & 9,472 & -58 & $1,585 \mathrm{E}-06$ & $9,999984150000 \mathrm{E}-01$ \\
\hline 0,1 & 0,546 & 19,487 & -45 & $3,162 \mathrm{E}-05$ & $9,999683800000 \mathrm{E}-01$ \\
\hline 0,05 & 1,106 & 39,494 & -36 & $2,512 \mathrm{E}-04$ & $9,997488113568 \mathrm{E}-01$ \\
\hline 0,02 & 2,786 & 99,497 & -25 & $3,162 \mathrm{E}-03$ & $9,968380000000 \mathrm{E}-01$ \\
\hline 0,01 & 5,586 & 199,499 & -18 & $1,585 \mathrm{E}-02$ & $9,841500000000 \mathrm{E}-01$ \\
\hline 0,005 & 11,186 & 399,499 & -16 & $2,512 \mathrm{E}-02$ & $9,748800000000 \mathrm{E}-01$ \\
\hline 0,002 & 27,986 & 999,500 & -10 & $1,000 \mathrm{E}-01$ & $9,000000000000 \mathrm{E}-01$ \\
\hline 0,001 & 55,986 & 1999,500 & -5 & $3,311 \mathrm{E}-01$ & $6,689000000000 \mathrm{E}-01$ \\
\hline 0,0005 & 111,986 & 3999,500 & -1 & $7,943 \mathrm{E}-01$ & $2,057000000000 \mathrm{E}-01$ \\
\hline 0,0004 & 139,986 & 4999,500 & $-0,5$ & $8,872 \mathrm{E}-01$ & $1,128000000000 \mathrm{E}-01$ \\
\hline 0,0003 & 186,653 & 6666,167 & $-0,3$ & $9,397 \mathrm{E}-01$ & $0,603000000000 \mathrm{E}-01$ \\
\hline
\end{tabular}

Table 1. Values of SE from CST simulations depending on the $S_{V}$

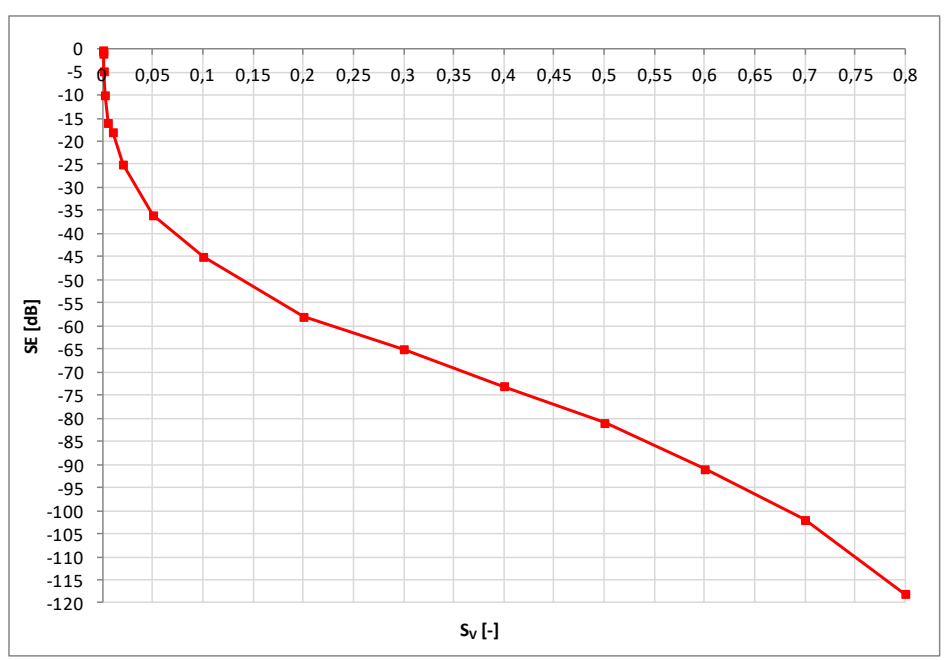

Fig. 8. Graphical representation of SE and $S_{V}$ dependencies from table 1 
The simulations were performed only up to $S_{V}=0.8$, which means $80 \%$ of the fiber content in the simulated fabric structure. Further simulations would not be relevant as the measurement approaches the noise level, which ranges from 113 to $-130 \mathrm{~dB}$ according to the measurement method.

\subsection{Calculation of transmission and reflection performance parameters}

The electromagnetic wave falls on the surface of the fabric sample of a given thickness $t$. Part of the energy is reflected $\left(P W R_{R}\right)$ from the surface, part of the energy is absorbed $\left(P W R_{D}\right)$ and the remaining energy passes through $\left(P W R_{T}\right)$ [9]. In general [1]:

$$
1=P W R_{R}+P W R_{D}+P W R_{T} \quad[-]
$$

The assumption for the above problem is the loss-free case where $P W R_{D}=0$. Then $P W R_{T}$ and $P W R_{R}$ are [1]:

$$
P W R_{T}=|T|^{2} \quad[-] \quad \text { and } \quad P W R_{R}=|R|^{2} \quad[-]
$$

where

$$
|T|=10^{\frac{-S E}{20}} \quad[-] \quad \text { and } \quad|R|^{2}=1-|T|^{2} \quad[-]
$$

The resulting $P W R_{T}$ and $P W R_{R}$ values obtained from the SE simulations are shown in tab. 1.

\subsection{Determining the percolation threshold of the composite model}

A jumping change from 0 to 1 for $P W R_{T}$ and 1 to 0 for $P W R_{R}$ is sought. The $S_{V}$ value at which these large jump changes occur can be considered as the assumed percolation threshold. The significance of this value is mainly in the field of electrically conductive materials for antistatic purposes, protection materials for electronics and electrotechnics.

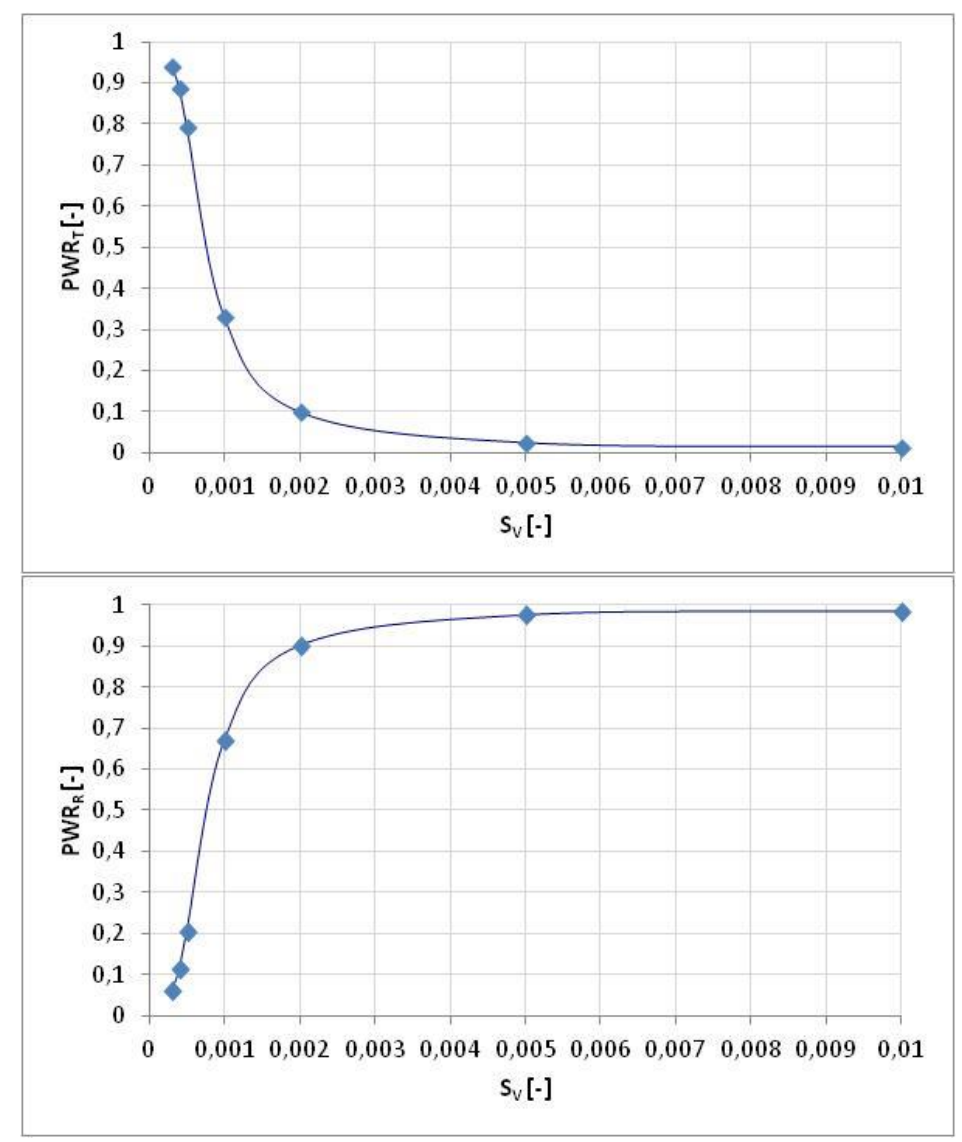

Fig. 9. Dependency of $P W R_{T}$ and $P W R_{R}$ on $S_{V}$ 


\section{Conclusion}

A metallized fabric from LORIX was chosen as a suitable composite material. This is a fabric with long copper-plated polyamide fibers designated by PBN-II-1.3-Cu-Sn. In order to verify its shielding efficiency, measurements were made on coaxial lines for a frequency range of 1 to $10 \mathrm{GHz}$. Comparison of the shielding effects of the measured sample supplied by the manufacturer and obtained by the measurements was performed only for the $1 \mathrm{GHz}$ frequency due to the known manufacturer's data for this sample. The shielding efficiency was measured at a given $1 \mathrm{GHz}$ frequency of $-87 \mathrm{~dB}$. The manufacturer supplied a lower value of $-75 \mathrm{~dB}$. It does not need to be a false result. It may be an indication of the real shielding efficiency of the sample. It can be assumed that the manufacturer supplied the minimum guaranteed shielding efficiency. Furthermore, for the theoretical determination of the percolation threshold of the composite sample, the simulation of the shielding effect of the fabric was based on the surface concentration of the fiber in its structure. Simulations were performed in a CST Microwave Studio to $80 \%$ fiber concentration in a simulated fabric structure. At this value, the fabric achieves nearly $-120 \mathrm{~dB}$, so further simulations leading in this direction would not be significant in the case of real measurements, given the dynamic range of the device used. Transmission and reflection parameters were determined by calculation. Determining the percolation threshold was made from the plot of the transmission and/or reflection parameters depending on the surface fiber concentration in the fabric. A jumping change from 0 to 1 for $P W R_{T}$ and 1 to 0 for $P W R_{R}$ was sought. The percolation threshold was determined to $0.03 \%$ of the fiber content in the simulated model fabric sample.

The suitability of the method used to determine the percolation threshold will be discussed in future work which will focus on determining the percolation threshold from the AC conductivity dependence to the concentration of the metallic fibers of the composite material. The selected frequency range will be determined in correlation of usage of a composite material.

\section{References}

[1] Paul, C. R. (2006). Introduction to Electromagnetic Compatibility. New Jersey: John Wiley \& Sons, Inc., 2006. ISBN 0-471-75500-1.

[2] Volski, V.; Aerts, W.; Vasylchenko, O. \& Vandenbosch, G. (2006). Analysis of Composite Textiles Filled with Arbitrarily Oriented Conducting Fibers using a Periodic Model for Crossed Strips. Proceedings of International Conference on Mathematical Methods in Electromagnetic Theory, Ukraine, Kharkiv, 2006, pp. 58-63. ISBN 14244-0490-8.

[3] Wieckowski, T. W. \& Janukiewicz, J. M. (2006). Methods for Evaluating the Shielding Effectiveness of Textiles. Fibres \& Textiles in Eastern, 2006, Vol. 14, No. 5, pp. 18-22. ISSN 1230-3666.

[4] Youngs, I. J. (2007). The Shielding Effectiveness of Composite Media - a Parametric Analysis Supporting an Engineering Perspective. IEEE Transactions on Dielectrics and Electrical Insulation, 2007, Vol. 14, No. 5, pp. 11451153. ISSN 1070-9878.

[5] Svačina, J. (2002). Electromagnetic Compatibility: Lectures. Czech Republic, Brno: Brno University of Technology, 2002. ISBN 80-214-1573-8.

[6] Michna, Š. \& al. (2007). Aluminium Materials and Technologies from A to Z. Slovakia, Prešov: Adin, s.r.o., 2007. ISBN 978-80-89244-18-8.

[7] Dřínovský, J.; Kejík, Z. (2009). Electromagnetic Shielding Efficiency Measurement of Composite Materials. Measurement Science Review, 2009, Vol. 9, No. 4, pp. 109-112.

[8] Lorix Ltd. (2011). Metallised textile. Available http://www.lorix.shp.hu/hpc/web.php?a=lorix\&o=950XGqatYS: 2011-12-19.

[9] Vojtěch, L. a Hájek, J. (2010). Measurement of Electromagnetic Shielding Efficiency of Planar Textiles in Frequency Range $100 \mathrm{kHz}$ - 1,5 GHz. Access Server, 2010. ISSN 1214-9675.

[10] Vilčáková, J. (2007). Electrical and Magnetic Properties of Polymer Composites: Habilitation thesis summary. Czech Republic, Zlín: Tomas Bata University in Zlín, 2007. ISBN 978-80-7318-519-0.

[11] Michna, Š. (2008). Composite Materials. Available from: http://www.stefanmichna.com: 2011-11-18.

[12] Skočík, P.; Goňa, S. \& Křesálek, V. (2011). Fullwave Modelling of Shielding Effectiveness of Metalized Textiles, Annals of DAAAM for 2011 \& Proceedings of the 22nd International DAAAM Symposium, 23-26th November 2011, Vienna, Austria, Volume 22, No. 1, ISSN 1726-9679, ISBN 978-3-901509-83-4, Katalinic, B. (Ed.), pp. 01010102, Published by DAAAM International Vienna, Vienna.

[13] Ř́mská, Z.; Křesálek, V. \& Špaček, J. (2002). AC Conductivity of Carbon Fiber - Polymer Matrix. Polymer Composites, 2002, Vol. 23, No. 1, pp. 95-103. ISSN 0272-8397. 